Communication et organisation

42 | 2012

La communication, dimension oubliée de l'intelligence économique

\title{
Un outil de décryptage de contenus manipulatoires : cas des groupes islamistes radicaux
}

Franck Bulinge

(2) OpenEdition

Journals

Édition électronique

URL : http://journals.openedition.org/communicationorganisation/3938

DOI : 10.4000/communicationorganisation.3938

ISSN : 1775-3546

Éditeur

Presses universitaires de Bordeaux

Édition imprimée

Date de publication : 1 décembre 2012

Pagination : 175-190

ISBN : 978-2-86781-772-4

ISSN : 1168-5549

\section{Référence électronique}

Franck Bulinge, « Un outil de décryptage de contenus manipulatoires : cas des groupes islamistes

radicaux », Communication et organisation [En ligne], 42 | 2012, mis en ligne le 01 décembre 2013, consulté le 01 mai 2019. URL : http://journals.openedition.org/communicationorganisation/3938 ;

DOI : 10.4000/communicationorganisation.3938 


\title{
Un outil de décryptage de contenus manipulatoires : cas des groupes islamistes radicaux
}

\author{
Franck Bulinge ${ }^{1}$
}

En mars 2012, Mohamed Merah, un Toulousain de vingt-trois ans, proche du milieu salafiste, commet une série de meurtres au nom d'un jihad qu'il semble mener seul. L'enquête nous apprend notamment que Merah tentait de rallier son entourage en l'invitant à regarder des vidéos jihadistes téléchargées sur Internet. Cette affaire remet en exergue la question récurrente que se posent les services de sécurité sur rôle d'Internet dans les stratégies de communication d'acteurs considérés comme une menace pour la sécurité nationale (SGDN, 2006 ; Mallet, 2008).

Cette recherche, conduite de novembre 2008 à avril 2009, visait à identifier les techniques de manipulation utilisées dans les contenus multimédias ainsi que leurs effets d'exposition potentiels sur les internautes. Dans un premier temps, nous avons passé en revue la littérature afin de définir les différents concepts, méthodes et techniques de manipulation qui peuvent être utilisés volontairement ou non par les auteurs. À partir de ces éléments, nous avons élaboré une grille d'analyse permettant de caractériser les contenus en fonction de leur niveau de risque et de leur effet d'exposition possible sur un public dont nous avions préalablement identifié les vulnérabilités. Enfin, nous avons soumis une douzaine de supports au crible de cette grille.

\section{Contexte théorique et hypothèses de recherche}

\section{Influence, manipulation, subversion : hypothèse d'un processus}

Il semble impossible qu'un individu issu d'un système socioculturel républicain et laïque puisse supporter spontanément des actes insoutenables tels que ceux véhiculés sur certains sites islamistes. Que dire des individus qui s'en délectent et les conseillent à leur entourage, à l'instar de Mohamed Merah ? De cette question émerge l'intuition d'une logique acceptation-légitimationappropriation sans laquelle les contenus les plus choquants seraient rejetés,

1 Franck Bulinge est professeur en management de l'information et de la communication à l'Institut supérieur du commerce de Paris, et chercheur au laboratoire I3M de l'université de Toulon ; franck.bulinge@iscparis.com 
ce qui, comme le démontre le cas Merah, n'est pas systématique. Or, comme le soulignent Akmouche et Hemery (2008), "statistiquement, pour rallier un djihadiste, il faut démarcher, en moyenne, 45000 personnes ». En ce sens, internet constitue un véritable espace de propagande (Thomas, 2008) voire de recrutement.

Notre recherche repose sur l'hypothèse d'un processus d'influence, manipulation, subversion (IMS) pouvant conduire certains individus à s'appuyer sur ces contenus pour un éventuel passage à l'acte terroriste. À travers ce processus, l'internaute cheminerait progressivement selon une trajectoire souhaitée par le manipulateur, à la fois acteur et victime d'un engrenage psychologique conduisant à son engagement dans une logique déviante.

Nous allons à présent définir ces différentes notions :

- L'influence vise à infléchir le jugement, à faire adhérer librement à une idée de manière non contraignante. Pour l'essentiel, l'influence recourt aux techniques d'information et d'argumentation en respectant le libre arbitre de l'influencé.

- La manipulation vise l'aliénation du jugement et du comportement d'une personne ou d'un groupe conformément à une attente (Breton, 2000), par le biais de techniques psychologiques que d'Almeida (2003) associe à la ruse et à la tromperie. La manipulation implique la perte du libre arbitre, elle n'est pas une finalité mais un moyen de parvenir à un objectif.

- La subversion, définie par le Petit Robert comme un "bouleversement, renversement de l'ordre établi, des idées et des valeurs reçues ", est généralement considérée comme une action de masse contre le pouvoir en place (Mucchielli, 1976). Toutefois, suivant Tchakhotine (1952) critiquant Le Bon (1895), nous considérons, que le processus la subversion est un processus dont on ne peut éluder la dimension individuelle. Dans le cadre de cette étude, la subversion est ainsi entendue comme le moment où un individu bascule d'un système de valeur à un autre (exemple : conversion religieuse) sous l'effet d'une manipulation.

- Le passage à l'acte est la mise en action de la violence terroriste. Castel (2011) évoque le "paradigme d'une violence inopinée, incoercible et catastrophique ", et le définit comme le "cheminement souterrain d'une passion", soulignant la complexité d'un geste sous-tendu par une intention. Le livre blanc sur la sécurité intérieure (SGDN, 2006) constate que « même si le passage à l'acte résulte toujours, en dernière instance, d'une décision individuelle mûrie solitairement, il est quasiment toujours exécuté dans un cadre collectif, surtout s'il s'accompagne du suicide de ses auteurs. » (LB, 2006). Ainsi, le passage à l'acte terroriste résulterait de l'appropriation puis de l'incarnation individuelle d'une violence collective nihiliste (Neyrat, 2009) conduisant au don symbolique de la mort (Baudrillard, 2002).

Notion d'effet d'exposition et engrenage manipulatoire 
Selon notre hypothèse, le fait de naviguer sur des sites à contenus manipulatoires pourrait entraîner certains internautes dans le filet tendu par leurs auteurs, ce qui suppose de leur part une stratégie globale déployée en réseaux.

En théorie, il s'agirait de répondre à la question : quel est le but poursuivi ? Les manuels de guerre psychologique évoquent dans ce cas la notion d'effet final recherché (EFR). Toutefois, cette expression suppose d'établir la réalité d'une intention, ce qui, pour un simple observateur et contre toute évidence, est objectivement impossible. C'est pourquoi nous préférons le terme " effet d'exposition possible » (EEP) qui traduit une conséquence possible de l'exposition individuelle à un contenu. On identifie cinq EEP qui correspondent aux différents stades IMS :

- La sensibilisation : objectif théoriquement visé par une stratégie d'influence. Il s'agit essentiellement d'informer et d'argumenter en vue d'instaurer un état de tolérance à l'égard d'un point de vue ou d'un système de valeurs différent. Dans le cas des islamistes, l'objectif consisterait à jouer sur les perceptions dans le but de neutraliser les facteurs anxiogènes (charia, salafisme, terrorisme) favorisant les situations de rejet (islamophobie).

- Le conditionnement : phase d'amorçage cognitif qui marque le début du stade de manipulation. Pour d'Almeida (2002), « la notion de conditionnement résume cette idée double : à la fois l'existence d'une phase précédant la demande et l'obtention de l'action, et l'entretien d'un état d'esprit garantissant le consentement du manipulé». Pour Alex Mucchielli (2006), "la propagande "de conditionnement" reste une référence à laquelle les divers fanatiques du monde reviennent toujours. Les idées de Tchakbotine sont encore appliquées [...] Elles prennent leurs sources dans la réflexologie de Pavlov [...]».

- L'endoctrinement : phase durant laquelle un individu se soumet à l'acceptation et à l'apprentissage de la doctrine (ici jihadiste). Il adhère aux valeurs et croyances qui lui sont inculquées et tourne le dos à son entourage. Cette phase marque le passage au stade de subversion. Lindividu s'enferme dans une logique mentale sectaire, selon le processus d'idéalisation décrit par Casoni et Brunet (2003), qui le prépare à entrer dans la cellule terroriste réelle ou virtuelle, laquelle lui donnera une nouvelle identité (phénomène de renaissance initiatique).

- L'embrigadement : c'est la phase durant laquelle l'individu intègre le groupe réel ou symbolique dont il s'approprie les gestes, les paroles et les comportements. D'Almeida l'associe à l'endoctrinement, mais nous ne partageons pas cette vision. En effet, alors que l'endoctrinement crée les conditions intellectuelles de la subversion, l'embrigadement est un acte engageant qui précède l'action.

- L'incitation au passage à l'acte : pour être identifié comme tel, cet EEP doit selon nous résulter d'un contenu explicite pouvant encourager et potentialiser le passage à l'acte. Par exemple, la diffusion d'une méthode de fabrication 
d'engins explosifs relève de l'embrigadement. Elle est une incitation au passage à l'acte si la cible est explicitement désignée (les Juifs, les Croisés).

\section{La question des vulnérabilités individuelles}

Analysant les profils de différents Européens convertis et morts en kamikazes, Landau (2008) conclut à l'impossibilité de profiler un futur jihadiste. Il semble par ailleurs impossible de dissocier le cheminement d'un " possible terroriste » de l'environnement social dans lequel il évolue.

Selon Abgrall (2003), " le manipulé idéal pour le manipulateur est un individu qui éprouve un sentiment d'incomplétude, né de sa non-appartenance (réelle ou supposée) à un groupe déterminé. Cette non-appartenance crée un vide affectif, existentiel dans les cas les plus extrêmes. De cette souffrance, surgit un désir irraisonné de se conformer au modèle social envié. » La littérature académique et militaire (Francart, 2000 ; Joule \& Beauvois, 2002 ; Guéguen, 2003 ; Cialdini, 2004 ; Desmaretz, 2004 ; Caramello, 2008), permet de dégager une typologie des vulnérabilités résumées dans le tableau suivant :

\begin{tabular}{|l|c|}
\hline \multirow{5}{*}{ Vulnérabilités psychoaffectives } & Besoin d'affection \\
Besoin d'appartenance \\
Besoin de reconnaissance \\
Émotivité, hypersensibilité \\
Empathie \\
Frustrations \\
Idéalisme \\
Manque de confiance en soi \\
Naïveté \\
Paranoïa \\
Perte de repères \\
Rébellion, refus de la société \\
\hline Vulnérabilités cognitives & Sensibilité à l'engagement (incapacité au refus) \\
Sentiment de solitude, d'abandon, d'infériorité \\
Soumission excessive à l'autorité
\end{tabular}

Tableau $n^{\circ} 1$ - Essai de typologie des vulnérabilités 
Nous pensons pour notre part qu'il est improbable de dresser un «portrait prédictif » de l'individu vulnérable. Toutefois, certains individus, en raison des éléments dominants de leur personnalité, et lorsqu'ils souffrent d'isolement et de désespérance, constituent a priori des proies idéales pour les manipulateurs. On cite souvent l'exemple des prisons où seraient recrutés de futurs jihadistes, mais cet état de solitude peut aussi bien concerner des personnes libres.

Notre cible type serait un internaute qui évolue dans un espace virtuel, une bulle dans laquelle sa relation aux autres est dématérialisée. Alors que l'absence d'interaction physique suggère une altération de la communication, Courbet et al. (2008) montrent que la médiatisation via un écran d'ordinateur, tout comme les relations asynchrones (chat, forums, e-mails), n'empêchent pas d'exercer un pouvoir d'influence ou de manipulation. L'objectif du manipulateur serait donc d'atteindre l'internaute dans cette bulle où il se sentirait paradoxalement en sécurité derrière son anonymat. Bien qu'il soit censé connaître les risques de l'Internet, l'internaute, lorsqu'il pénètre certains cyberterritoires, se comporterait dès lors comme la proie qui se désaltère au milieu des prédateurs. La maîtrise de l'espace informationnel dépend de sa vigilance et de ses capacités cognitives (perception et analyse). Mais la vigilance peut être trompée par des artifices visuels et sonores. De même, sa capacité d'analyse (libre arbitre) peut être affectée par des chocs émotionnels ou des biais cognitifs que nous avons énumérés et définis par ailleurs (Bulinge, 2010). En théorie, lorsque ces vulnérabilités sont confrontées à des techniques d'influence et de manipulation, elles peuvent générer un phénomène IMS. Nous allons à présent passer en revue ces techniques.

\section{Revue des techniques de manipulation}

Breton (2000) et Abgrall (2003) identifient deux grandes familles de manipulation. La première consiste à jouer avec les affects du manipulé, alors que la seconde vise ses capacités cognitives. Les auteurs précisent que l'une et l'autre sont inséparables et qu'elles agissent de concert sans qu'il soit parfois possible de les distinguer.

a) Manipulation affective

Il s'agit, pour Abgrall (2003), de créer un courant affectif entre le manipulateur et sa cible. Nous parlons de registres affectifs ou émotionnels qui vont de l'humour à la colère en passant par la peur, l'angoisse ou la tristesse. La manipulation opère sur la forme du message et repose sur une relation d'identification subjective (empathie, séduction, effet fusionnel). Il s'agit de déclencher des sentiments aux dépens d'une analyse rationnelle. Par effet de substitution, les sentiments prennent alors le pas sur le raisonnement dans la construction de l'opinion.

La manipulation peut être envisagée selon deux approches : 
- L'association d'éléments sensoriels soit comme support du message (nursing des sens, léthargie intellectuelle, sophronisation), soit comme stimuli euphorisants ou anxiogènes agissant directement sur les émotions (exemples : infrasons, succession rapide d'images provocant un état de stress particulier).

- L'amalgame affectif visant le transfert de sentiments entre deux éléments (exemple : association de l'image d'un homme politique avec des symboles sataniques).

Les techniques utilisées porteront par conséquent sur : l'usage d'artifices sensoriels visant l'esthétisation du message (contenu textuel, images, sons, musique) ; le pouvoir évocateur des symboles ; les effets fusionnels (identité, foi, ivresse et émotion collective), l'impact narratif (storytelling); l'association d'images, de texte et de sons, etc. Voici quelques exemples commentés :

- Esthétisation textuelle : mise en place d'un champ lexical et sémantique susceptible de séduire/apaiser/émouvoir le sujet. Il peut s'agir d'une forme de lyrisme, d'effets poétiques, de phrases emphatiques, voire d'un style produisant un effet agréable accrochant le lecteur. Remarque : l'esthétisation dépend de la culture du sujet : l'évocation du martyr dans un style romantique (flou gaussien, musique héroïque et discours empathique) ne sera pas perçue de la même manière par un aspirant jihadiste et par un analyste occidental.

- Effets visuels : esthétisation et trucage susceptibles de générer des émotions : images d'enfants, de misère avec filtres de type gaussien (sentiment de pitié, d'empathie); répétition ou succession d'images anxiogènes produisant des effets d'accélération et de saturation; techniques subliminales (Pessiglione et al., 2008) ; couleurs et formes signifiantes (rouge, feu, armes).

- Effets sonores et musicaux : musiques et sons induisant des sensations (bien-être, bonheur, angoisse, violence) susceptibles d'altérer l'état de vigilance du sujet. Les travaux de Bigand (2008) montrent l'effet de la musique sur les émotions (colère/peur, tristesse, gaieté et sérénité) qui agissent sur les schémas cognitifs, l'affect et le corps.

- Storytelling : narration visant à replacer artificiellement la cible dans un contexte émotionnel favorable (Salmon, 2007).

b) Manipulation cognitive

La manipulation cognitive vise à altérer le processus de raisonnement à l'aide de subterfuges (fausses informations, rhétorique). Philippe Breton (2000) distingue deux grandes techniques :

- Le cadrage manipulateur consiste à utiliser des éléments connus et acceptés par l'interlocuteur et à les réordonner d'une façon telle qu'il ne peut guère s'opposer à leur acceptation.

- L'amalgame cognitif consiste à proposer un cadrage des faits en y associant des éléments dont l'apport sera en lui-même convaincant. Il vise à créer une chaîne de causalités apparentes qui en réalité, est infondée. L'exemple typique, utilisé par les publicitaires, consiste à associer un concept à des mots 
considérés comme «bons » ou « mauvais » en fonction de l'orientation qu'on souhaite donner.

Les tableaux suivants présentent une synthèse des techniques de manipulation affective et cognitive relevées dans la littérature et/ou identifiées de manière empirique dans les contenus Internet :

\begin{tabular}{|c|c|c|c|}
\hline & Méthode & Effet recherché & Techniques \\
\hline \multirow{4}{*}{$\begin{array}{l}\text { Esthétisation } \\
\& \\
\text { manipulation } \\
\text { sensorielle }\end{array}$} & Nursing des sens & $\begin{array}{l}\text { Relaxation, } \\
\text { sophronisation, état } \\
\text { pré-hypnotique, } \\
\text { léthargie intellectuelle, } \\
\text { sentiment de } \\
\text { fusion, régression, } \\
\text { identification }\end{array}$ & $\begin{array}{l}\text { Images : cadrage ou recadrage, } \\
\text { effets de loupe (focalisation sur un } \\
\text { point), retouche (flous gaussiens, } \\
\text { couleurs accentuées, noir et blanc, } \\
\text { granulation), stéréotypes (enfants, } \\
\text { vieillards), choc des photos } \\
\text { (cadavres, scènes violentes) }\end{array}$ \\
\hline & \multirow{3}{*}{ Excitation } & \multirow{2}{*}{$\begin{array}{l}\text { Sentiments de peur, } \\
\text { tristesse, compassion, } \\
\text { confiance }\end{array}$} & $\begin{array}{l}\text { Sons : infrasons (sensation } \\
\text { d'angoisse), son aigus, déchirants } \\
\text { (stress), musique synchronisée sur } \\
\text { l'image ou le contexte }\end{array}$ \\
\hline & & & $\begin{array}{l}\text { Voix : masculine ou féminine, } \\
\text { d'enfants; lente, profonde, } \\
\text { hypnotique, utilisation d'effets } \\
\text { (écho, réverbération, crescendo) }\end{array}$ \\
\hline & & $\begin{array}{l}\text { Sensations de vertige, } \\
\text { d'élévation, de } \\
\text { transcendance, de stress, } \\
\text { d'angoisse, d'euphorie }\end{array}$ & $\begin{array}{l}\text { Textes : effets de style (lyrique, } \\
\text { religieux, proverbial, poétique), } \\
\text { choix du champ sémantique (le } \\
\text { «choc des mots», tonalité positive } \\
\text { ou négative, apaisante, angoissante } \\
\text { ou violente) }\end{array}$ \\
\hline \multirow{4}{*}{$\begin{array}{l}\text { Amalgame } \\
\text { affectif \& } \\
\text { cognitif }\end{array}$} & \multirow{4}{*}{ Associations } & $\begin{array}{l}\text { Effets anxiogènes, } \\
\text { stress et réponse } \\
\text { physiologique (pouls, } \\
\text { sudation, malaise, } \\
\text { nervosité) }\end{array}$ & $\begin{array}{l}\text { Enchaînement rapide et répété } \\
\text { d'images }\end{array}$ \\
\hline & & $\begin{array}{l}\text { Transfert de charge } \\
\text { affective d'un sujet/ } \\
\text { objet vers un autre }\end{array}$ & $\begin{array}{l}\text { Voix, images et sons associés : } \\
\text { exemple Bush+franc- } \\
\text { maçonnerie+sons et voix sataniques, } \\
\text { CIA+torture+hurlements }\end{array}$ \\
\hline & & $\begin{array}{l}\text { Activation de croyances } \\
\text { et sentiments primaires } \\
\text { (racisme, xénophobie, } \\
\text { superstitions) }\end{array}$ & $\begin{array}{l}\text { Symbolisme : universel (enfer, feu, } \\
\text { mort) ou spécifique à la culture } \\
\text { (croissant, kalashnikov, cheval, } \\
\text { svastika...) }\end{array}$ \\
\hline & & $\begin{array}{l}\text { Leviers psychologiques } \\
\text { (paranoïa, narcissisme) }\end{array}$ & $\begin{array}{l}\text { Storytelling: narration visant à } \\
\text { recontextualiser la perception ou } \\
\text { appelant des valeurs programmées } \\
\text { (patriotisme, héroïsme, martyr) }\end{array}$ \\
\hline
\end{tabular}




\begin{tabular}{|c|c|c|c|}
\hline & Méthode & Effet recherché & Techniques \\
\hline \multirow{10}{*}{$\begin{array}{c}\text { Cadrage } \\
\text { manipulateur }\end{array}$} & $\begin{array}{l}\text { Cadrage } \\
\text { menteur }\end{array}$ & $\begin{array}{l}\text { Altération du } \\
\text { jugement par } \\
\text { introduction } \\
\text { de données } \\
\text { fausses, truquées } \\
\text { ou tronquées } \\
\text { (désinformation) }\end{array}$ & $\begin{array}{l}\text { Truquage des contenus textuels, vocaux, sonores } \\
\text { et visuels (ajouts, suppressions, retouches, } \\
\text { décontextualisation, etc.) }\end{array}$ \\
\hline & \multirow{7}{*}{$\begin{array}{l}\text { Cadrage } \\
\text { abusif }\end{array}$} & \multirow{7}{*}{$\begin{array}{l}\text { Recherche } \\
\text { d'adhésion et de } \\
\text { conviction par } \\
\text { la manipulation } \\
\text { du langage et des } \\
\text { représentations } \\
\text { visant l'aliénation } \\
\text { du sens critique } \\
\text { (propagande) }\end{array}$} & $\begin{array}{l}\text { Ambigüités : usage de termes, d'images ou de } \\
\text { sons laissant un doute sur leur interprétation } \\
\text { possible. Usage de formules conditionnelles non } \\
\text { vérifiables }\end{array}$ \\
\hline & & & $\begin{array}{l}\text { Arguments d'autorité : références à des personnes } \\
\text { dont il est difficile de discuter l'autorité (Dieu, } \\
\text { l'expert, le scientifique) }\end{array}$ \\
\hline & & & $\begin{array}{l}\text { Répétitions : usage répété de termes, de sons ou } \\
\text { d'images. Vise à faire admettre le message comme } \\
\text { une évidence (Allah Akbar; 9/11; terrorisme) }\end{array}$ \\
\hline & & & $\begin{array}{l}\text { Mots piégés : emploi de mots renvoyant à } \\
\text { des représentations qualifiantes (louangeurs, } \\
\text { dépréciateurs, neutralisants, justifiants, } \\
\text { déresponsabilisants) exemples : solution finale ; } \\
\text { problème juif; terroriste ; moujahidine; taliban ; } \\
\text { dommage collatéral ; frappe chirurgicale }\end{array}$ \\
\hline & & & $\begin{array}{l}\text { Rails mentaux : usage de représentations } \\
\text { totémiques ou tabous (révisionnisme, Shoa, } \\
\text { terroriste, Croisades, Bien et Mal,...) qui ferment } \\
\text { le cadre d'analyse }\end{array}$ \\
\hline & & & $\begin{array}{l}\text { Mythologie programmée : création d'un cadre } \\
\text { mythique dans lequel est enfermé l'esprit critique } \\
\text { de l'individu (exemples : sentiment national, } \\
\text { Mère patrie, défense de la foi, race supérieure, } \\
\text { élitisme...) }\end{array}$ \\
\hline & & & $\begin{array}{l}\text { Manipulation des perceptions : voir les } \\
\text { techniques d'esthétisation et de manipulation } \\
\text { sensorielle dans la manipulation des affects }\end{array}$ \\
\hline & \multirow[t]{2}{*}{$\begin{array}{l}\text { Cadrage } \\
\text { contraignant }\end{array}$} & \multirow{2}{*}{$\begin{array}{l}\text { Obtenir un } \\
\text { consentement } \\
\text { négocié en } \\
\text { procédant par } \\
\text { étapes (engrenage, } \\
\text { théorie de } \\
\text { l'engagement) }\end{array}$} & $\begin{array}{l}\text { Pied dans la portex: consiste dans un premier } \\
\text { temps à amorcer le sujet à l'aide d'une première } \\
\text { sollicitation pour laquelle la réponse ne nécessite } \\
\text { pas un engagement contraignant (consensus). Cet } \\
\text { accord obtenu sert de point d'appui pour faire } \\
\text { accepter une opinion plus contraignante (ancrage) }\end{array}$ \\
\hline & & & $\begin{array}{l}\text { Porte dans le nez: consiste à proposer une } \\
\text { première représentation inacceptable en vue de } \\
\text { rendre acceptable une seconde proposition. }\end{array}$ \\
\hline
\end{tabular}




\begin{tabular}{|c|c|c|c|}
\hline \multirow{4}{*}{$\begin{array}{c}\text { Amalgame } \\
\text { cognitif }\end{array}$} & \multirow{4}{*}{$\begin{array}{l}\text { Association } \\
\text { bétabloquante }\end{array}$} & \multirow{4}{*}{$\begin{array}{l}\text { Activation de } \\
\text { représentations } \\
\text { sociales fortes à } \\
\text { l'aide de mots } \\
\text { et de symboles } \\
\text { appelés leviers, } \\
\text { en vue de bloquer } \\
\text { le sens critique } \\
\text { (bétabloquants } \\
\text { mentaux) }\end{array}$} & $\begin{array}{l}\text { Leviers de vertu : appel aux valeurs (démocratie, } \\
\text { liberté, justice, indépendance, fraternité, égalité...) }\end{array}$ \\
\hline & & & $\begin{array}{l}\text { Leviers poisons : association de mots à } \\
\text { valence négative (guerre, massacre, fascisme, } \\
\text { révisionnisme, terrorisme) }\end{array}$ \\
\hline & & & $\begin{array}{l}\text { Leviers d'autorité : référence aux autorités } \\
\text { religieuses, scientifiques ou morales intouchables } \\
\text { (Dieu, Mahomet, le Pape, Gandhi, Mère Thérésa, } \\
\text { Einstein) }\end{array}$ \\
\hline & & & $\begin{array}{l}\text { Leviers de conformisme : appel à la solidarité, à } \\
\text { la pression émotionnelle et collective (l'Oumma, la } \\
\text { chrétienté, la terre promise, le Reich) }\end{array}$ \\
\hline
\end{tabular}

Tableau $n^{\circ} 3$ - Manipulation cognitive

\section{Méthode et outil d'analyse}

\section{Méthodologie d'analyse}

De notre modèle théorique, nous déduisons un cheminement méthodologique illustré par la figure $n^{\circ} 2$.

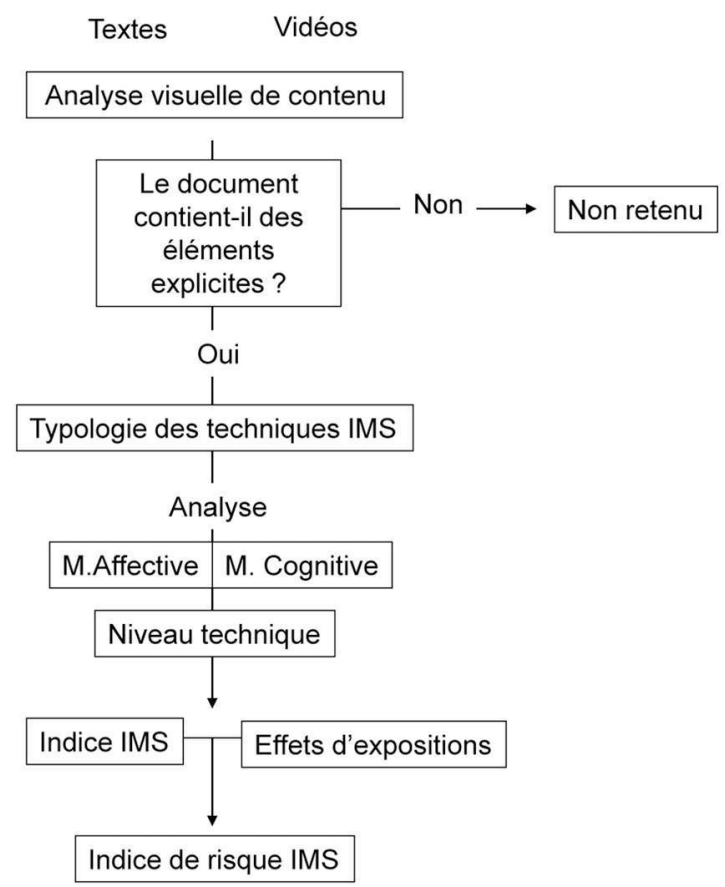

Figure $n^{\circ} 1$ - Méthodologie de traitement et d'analyse 
En premier lieu, nous explorons visuellement les contenus Internet (textes, images, vidéos). Si nous identifions des éléments significatifs, nous passons à la phase d'analyse et, en nous appuyant sur les typologies présentées plus haut, nous repérons les techniques utilisées. Nous mesurons alors la part de manipulation basée sur les affects (matrice émotionnelle), et celle basée sur l'intellect (matrice cognitive), puis nous évaluons le niveau technique du support. Cela nous permet de calculer un indice de manipulation (Indice IMS) ainsi que les effets d'expositions possibles. Enfin, nous en déduisons un indice de risque IMS.

Remarque : L'analyse débute dès la phase exploratoire, et sert aussi bien à repérer des techniques connues que d'éventuelles techniques non répertoriées. Il en découle une démarche à la fois déductive et inductive, puisque nous partons du modèle théorique que nous enrichissons en situation à l'aide de l'observation sur le terrain.

\section{Élaboration d'une matrice d'analyse}

Nous avons élaboré une grille d'analyse sur la base des éléments théoriques cités précédemment, et à partir des éléments identifiés empiriquement sur les objets Internet. Une série d'allers et retours entre supports théoriques et expérimentation nous a ensuite permis d'affiner le modèle, tant du point de vue de la mesure que de l'ergonomie.

Description de la matrice (figure $\mathrm{n}^{\circ} 2$ ) :

Elle se présente sous forme d'un tableau d'indicateurs au centre duquel l'opérateur dispose d'un espace de commentaires. Le choix d'un cadre bleu et d'un logo se justifie par la nécessité pour l'opérateur de conserver ses repères (charte graphique).

- La colonne "Manipulation psychosociale » représente les éléments d'analyse des manipulations affectives, culturelles, psychologiques et sociales. L'appel aux sentiments est traité de manière exhaustive afin de distinguer la nature des sentiments appelés, lesquels déterminent les EEP.

- La colonne "Manipulation cognitive », représente les éléments ayant un impact sur les capacités cognitives des sujets. Nous distinguons plus bas les techniques d'influence qui marquent l'entrée du sujet dans la zone IMS.

La colonne "Effet d'exposition possible » (EEP) permet d'évaluer les conséquences possibles d'une exposition individuelle.

L'indice technique résulte de l'évaluation faite par l'opérateur du degré de maîtrise technique du support et de son contenu. Il entre dans le calcul de l'indice IMS.

- L'indice IMS. Il s'agit d'un indice calculé à partir des éléments relevés sur les objets, sans préjuger des vulnérabilités des internautes, ni de l'intention réelle des auteurs.

Calcul de l'indice IMS : il correspond à la somme des éléments de manipulation relevés, multipliée par l'indice technique. Ce dernier influence, 
en effet, l'accessibilité du contenu, agissant comme un levier ou, au contraire, comme un frein à l'impact cognitif et affectif du contenu. Il est calculé sur une base 10 .

La formule retenue est la suivante :

IMS $=($ MAffect + MCog $) \times$ NTech $/ 20$

Calcul de l'indice de risque (IndRisq) : L'indice de risque donne une idée du niveau de risque que présente le contenu au regard des techniques IMS et de l'effet d'exposition possible. Il s'agit d'un indice relatif qui mise sur l'hypothèse d'un effet multiplicateur entre l'indice IMS et l'EEP. Calculé sur une base 10, il est obtenu en multipliant l'indice IMS par l'indice EEP. L'indice maximum étant de $10 \times 5$, soit 50 , on obtient la formule suivante :

IndRisq = IMS $\times$ EEP $\times 2 / 10$

\section{La phase expérimentale}

\section{Accès aux contenus islamistes sur Internet ${ }^{2}$}

Il est impossible de dénombrer les sites, blogs, forums islamistes sur le web. Une approche exhaustive était donc impossible. Nous avons par conséquent opté pour une approche simple de recherche par mots clés qui ressemble le plus possible à une logique d'internaute désireux de trouver des sites sur l'islam. On obtient une entrée sur le portail World of Islam. Ce portail attire l'attention du fait de sa vocation à renvoyer l'internaute vers d'autres sites référencés, parmi lesquels il est possible d'identifier des "sites innocents ", des sites propagandistes, enfin des sites " extrêmes " à contenus explicites (salafistes, qaïdistes, jihadistes). L'analyse réticulaire, réalisée à l'aide de la fonction Link+Url de Google, met en évidence de sites/blogs faisant office de hubs.

\section{Typologie des contenus}

On distingue principalement quatre types de contenus : textes, images, chants, vidéos. Leur analyse montre une diversité directement influencée par les courants représentés. Certains sites, notamment qaïdistes, présentent des contenus essentiellement textuels à vocation doctrinale (Kepel, 2005) ou pédagogique (fabrication de bombes, utilisation d'armes). Ils sont généralement accompagnés de musique ou de chants tandis qu'une partie du décryptage symbolique consiste à étudier l'imagerie qui les accompagnent (logos, fonds d'écran). On trouve également des sites de commerce en ligne qui vendent des effets vestimentaires salafistes.

Les vidéos occupent une place prépondérante dans les stratégies de communication des islamistes. Leur diversité nécessiterait à elle seule une typologie : prêche, préparation d'explosifs, démonstrations d'entraînement,

2 Les sites islamistes sont extrêmement volatiles, leur durée de vie est rendue très courte par leur fréquente migration. 
attentats, testaments filmés, scènes de combat, attentats suicides, exécutions, etc. Certaines, particulièrement violentes et barbares, sont insoutenables et choquantes y compris pour un professionnel du renseignement.

Exemple d'analyse d'un site avec la matrice IMS

Il concerne le site jihadiste Bagdad Snipper (figure n ${ }^{\circ}$ ). L'indice de risque, mesuré à partir d'un indice IMS 8 et d'un EEP maximum (incitation au passage à l'acte), est de 8/10. Cela signifie que ce site et son contenu sont de nature à inciter un public vulnérable à l'action terroriste.

\begin{tabular}{|c|c|c|c|c|c|}
\hline \multicolumn{6}{|c|}{ FICHE D'ANALYSE } \\
\hline & & $\mathrm{N}^{\circ}$ & $15 / 2008$ & & Courant \\
\hline & & Référence BDD & Bagdad Snipper & & Qaidiste \\
\hline & & \begin{tabular}{|l|l|} 
Type \\
\end{tabular} & Site Web2.0 & $\mathrm{x}$ & Jihadiste régional \\
\hline 3.D & & \begin{tabular}{|l} 
Description \\
\end{tabular} & Site Bagdad Snipper & & Salafiste \\
\hline & & \begin{tabular}{|l|l} 
Date de capture \\
\end{tabular} & & & Islamiste \\
\hline & & Source & www.bagdadsniper.net & & \\
\hline & & \multicolumn{2}{|l|}{ Traitant } & & \\
\hline \multicolumn{2}{|c|}{ Manipulation psychoaffective } & \multirow{18}{*}{\multicolumn{2}{|c|}{$\begin{array}{l}\text { Le site du Bagdad Snipper est emblématique de la stratégie de } \\
\text { communication des jihadistes irakiens. Jouant sur le registre } \\
\text { de l'héroissation, le site crée le culte du BS. Les journalistes } \\
\text { sont invités à le contacter ainsi que les internautes de toutes } \\
\text { nationalités qui peuvent lui laisser un mess sage. Le jeu de } \\
\text { l'interactivité événementielle et le style moderne et dépouillé } \\
\text { le rendent particulièrement attractif. Il est en ce sens très } \\
\text { différent des sites islamistes habituels. Les vidéos } \\
\text { disponibles sont bien construites. L'analyse de trafic montre } \\
\text { une fréquentation significative dominée par les internautes } \\
\text { algériens. }\end{array}$}} & \multicolumn{2}{|r|}{ Manipulation cognitive } \\
\hline \multicolumn{2}{|l|}{ Esthétisation du message } & & & 1 & Voix/chants hypnotiques \\
\hline Esthétisation visuelle & 1 & & & 1 & $\begin{array}{l}\text { Voix/chants hypnotiques } \\
\text { Trucages visuels ou sonores }\end{array}$ \\
\hline $\begin{array}{l}\text { Esthétisation sonore } \\
\text { Storytelling }\end{array}$ & \multirow{2}{*}{$\frac{1}{1}$} & & & 1 & Enchaînement d'images \\
\hline \multirow{2}{*}{\begin{tabular}{|l} 
Storytelling \\
Associations symboliques
\end{tabular}} & & & & 1 & \\
\hline \multirow{2}{*}{\multicolumn{2}{|c|}{\begin{tabular}{|l|} 
Associations symboliques \\
Références identitaires \\
\end{tabular}}} & & & 1 & $\begin{array}{l}\text { Amalgames sonsttexte+Images } \\
\text { Mythologie programmée }\end{array}$ \\
\hline & & & & 1 & Rails mentaux \\
\hline \multirow{2}{*}{\multicolumn{2}{|c|}{ Conspirationnisme }} & & & 1 & Arguments d'autorité \\
\hline & & & & 1 & Leviers bétabloquants \\
\hline \multirow{2}{*}{\multicolumn{2}{|c|}{ Activation de sentiments }} & & & 1 & Mots et images explicites \\
\hline & Violence & & & 1 & Mots piégés \\
\hline \multicolumn{2}{|l|}{ Vengeance } & & & 1 & Informations tronquées \\
\hline \multicolumn{2}{|l|}{ Haine } & & & 1 & \begin{tabular}{|l|} 
Ambiguités \\
\end{tabular} \\
\hline \multicolumn{2}{|l|}{ Racisme } & & & 1 & Répétitions \\
\hline \multirow{2}{*}{\multicolumn{2}{|c|}{\begin{tabular}{|l} 
Colère/Dégoût \\
Angoisse/Peur \\
\end{tabular}}} & & & & Influence \\
\hline & & & & 1 & Suggestion \\
\hline Injustice & 0 & & & 1 & Argumentation \\
\hline Tristesse/Compassion & 0 & & & 1 & Information \\
\hline Total & 9 & Effet d'ex & sition nossible & 16 & Total \\
\hline Niveau technique & & Incitation à l'action violente & & & Indice IMS \\
\hline & & Embrigadement & & & \\
\hline & & Subversion & & & \\
\hline 10 & & \begin{tabular}{|l|} 
Endoctrinement \\
\end{tabular} & & & 8 \\
\hline & & \begin{tabular}{|l|} 
Conditionnement \\
\end{tabular} & & & \\
\hline
\end{tabular}

Figure $n^{\circ} 2$ - Matrice d'analyse IMS appliquée à un site multimédia

\section{Analyse comparative}

La matrice IMS permet par ailleurs de faire une analyse comparative des données recueillies sur les sites islamistes y compris francophones. Le tableau ci-dessous permet d'évaluer le niveau général de risque lié à une exposition régulière. Chaque indice est calculé sur une base 10 .

Ici on constate que le niveau technique est au-dessus de la moyenne, les EEP atteignant un niveau d'embrigadement. Cependant, les indices de manipulation ne dépassent pas la moyenne (4,8 et 4,3), de même pour l'indice moyen de risque qui est globalement bas (3 sur 10).

Le site du Bagdad Sniper est, de loin, celui qui présente un risque réel. De fait, il s'agit d'un site djihadiste « incontournable » montrant comment abattre les soldats américains en Irak, à travers un mix de textes de propagande et de 


\begin{tabular}{|l|c|c|c|c|c|c|}
\cline { 2 - 7 } \multicolumn{1}{c|}{} & Ntech & Maffect & Mcog & IMS & EEP & IndRisq \\
\hline Bagdad Sniper & $\mathbf{1 0}$ & 6 & $\mathbf{1 0}$ & 8 & 5 & $\mathbf{8}$ \\
Prisoner of Joice & 8 & 7 & 4 & 5 & 4 & $\mathbf{4}$ \\
Al Boraq & $\mathbf{1 0}$ & 6 & 3 & 4 & 4 & $\mathbf{4}$ \\
Voix des Opprimés & 8 & 5 & 5 & 5 & 3 & $\mathbf{3}$ \\
Al Ansar alhaqq & 5 & 5 & 6 & 5 & 4 & $\mathbf{4}$ \\
Iraq Islamic Army & 6 & 6 & 3 & 4 & 5 & $\mathbf{4}$ \\
Ribaat & 8 & 5 & 4 & 5 & 3 & $\mathbf{3}$ \\
Jihad Reform Front & 2 & 5 & 4 & 5 & 5 & $\mathbf{5}$ \\
Minbar SOS & 4 & 5 & 4 & 4 & 3 & $\mathbf{3}$ \\
Khilafat & 5 & 1 & 4 & 3 & 2 & $\mathbf{1}$ \\
Sentiers itinérants & 2 & 2 & 3 & 2 & 5 & $\mathbf{2}$ \\
Projihab & 7 & 4 & 1 & 2 & 2 & $\mathbf{1}$ \\
\hline Niveaux moyens/10 & $\mathbf{6 , 3}$ & $\mathbf{4 , 8}$ & $\mathbf{4 , 3}$ & $\mathbf{4 , 4}$ & $\mathbf{3 , 8}$ & $\mathbf{3}$ \\
\hline
\end{tabular}

Tableau $\mathrm{n}^{\circ} 4$ - Analyse des risques IMS sur 12 sites islamistes

vidéos mises en scène et techniquement maîtrisées. Le site est proposé en plusieurs langues (dont le français et l'allemand), l'incitation étant d'autant plus forte qu'elle est réalisable partout où il y a des militaires ${ }^{3}$.

\section{Portée et limites de l'outil}

\section{Un outil collaboratif}

La matrice d'analyse IMS permet de guider les opérateurs dans leur travail, et notamment de visualiser systématiquement et rapidement l'ensemble des paramètres à partir desquels ils peuvent évaluer un contenu textuel ou multimédia, tout en offrant un cadre protecteur compte tenu du risque, même minime, d'être influencé par le contenu des objets analysés.

Elle offre simultanément au décideur une représentation synthétique explicite facilitant la prise de décision. Elle constitue ainsi un outil de partage d'information qui permet d'envisager une collaboration analyste-décideur simple et efficace, basée sur un langage commun, une procédure de travail standardisée garantissant l'exhaustivité et la neutralité des données.

\section{Limites}

Cette matrice est un instrument de mesure qui ne prédit pas un éventuel passage à l'acte, mais donne une estimation du risque potentiel de manipulation ainsi qu'une conséquence possible à l'exposition, quelle que soit l'intention des auteurs. De même elle ne permet pas de préjuger de l'état ou de l'attitude d'un internaute lorsqu'il visualise un objet potentiellement manipulateur. Bien que reposant sur des indices objectifs, la matrice ne permet pas de déduire l'objectif visé par les auteurs de l'objet Internet. Il revient à l'analyste d'évaluer si l'objet favorise le conditionnement, l'endoctrinement, l'embrigadement ou l'incitation à la violence, à partir des indices relevés dans les contenus. Il en est

3 En 2008, nous attirions l'attention des autorités des services de renseignement sur le risque réel de ce site. Au regard du drame de Toulouse, on peut se demander dans quelle mesure il a pu influencer Mohamed Merah. 
de même pour l'évaluation technique qui relève à ce stade d'une estimation, mais qui pourrait par la suite faire l'objet d'une mesure plus précise. Le mode de calcul de l'indice de risque IMS reste, à ce stade, totalement empirique, et nécessiterait d'être fixé à partir d'une expérimentation sur un échantillon de population significatif. La matrice ne tient pas compte de l'impact d'un contenu en termes de flux (nombre de visiteurs) et de propagation dans les réseaux (référencement), ainsi que des conditions d'environnement global (notamment la publicité faite par les médias). Il convient par conséquent d'utiliser des outils complémentaires tels que l'analyseur de trafic Alexa.com. Enfin, son utilisation nécessite une bonne maîtrise des éléments d'analyse de la part des opérateurs, ce qui suppose une formation préalable sur les concepts utilisés. Le développement d'une application plus élaborée permettrait d'inclure un système d'auto-apprentissage ainsi qu'une base de données illustrant par des exemples les techniques utilisées.

\section{Conclusion}

Cette recherche nous a permis d'explorer un sujet encore assez peu étudié, comme le montre la littérature plutôt éparse qu'il nous a fallu réunir. Le terrain d'expérimentation, par définition extrême, nous a été imposé, et, dans une certaine mesure, il constituait un risque d'instrumentalisation politique au regard des contenus étudiés. Il s'agissait, par conséquent, de les aborder en étant conscient d'une part, de la complexité de l'espace dans lequel s'inscrivent ces contenus, et, d'autre part, de l'impossible neutralité du chercheur face à certains contenus insoutenables. Nous avons donc élaboré un modèle dans des conditions extrêmes, alors même que le cadre théorique auquel nous nous sommes référé était issu de la psychologie expérimentale. Or, nous pensons qu'il est difficile de prédire les réactions d'internautes au regard de théories développées en laboratoire. Par ailleurs, bien que l'outil d'analyse IMS permette d'identifier des techniques de "guerre de l'information ", nous ne sommes pas en mesure d'affirmer que ces techniques aient été mises en œuvre sciemment. De fait, notre méthode repose sur l'observation et l'identification d'éléments que nous qualifions de techniques au regard de nos connaissances dans ce domaine. En aucun cas cela ne nous autorise à préjuger des connaissances et de la maitrise de ces techniques par les auteurs des contenus Internet. C'est pourquoi, dans ce cas précis, notre matrice vise moins l'évaluation d'une stratégie d'influence que la mesure d'un risque potentiel lié à une exposition.

L'outil développé dans cette recherche permet, on l'a vu, de contribuer à une analyse structurée et systématique des contenus manipulatoires identifiables dans les stratégies de communication de groupes extrémistes. Une autre partie de cette recherche a d'ailleurs montré des résultats comparables chez les néonazis. Son affinement et son enrichissement pourraient répondre à des problématiques d'analyse plus globales, dépassant largement le cadre de la défense et de la sécurité nationale. Les applications marketing sont 
ainsi envisageables, la matrice pouvant, par exemple, servir d'outil d'analyse comparative de campagnes publicitaires. En politique, on envisagera le décryptage et l'analyse comparative des discours. Dans le domaine de la presse, il contribuera à l'identification et à l'analyse d'éventuels phénomènes manipulatoires.

\section{BIBLIOGRAPHIE}

\section{Influence, manipulation, subversion}

ABGRALL J.-M., Tous manipulés, tous manipulateurs, Paris, First Éditions, 2003, $372 \mathrm{p}$.

ALMEIDA F., La manipulation, Paris, PUF, Que sais-je ?, 2002, 125 p.

BIGAND E., Les émotions musicales, Pour la Science, n³73, novembre 2008, p. $132-138$

BRETON P., La parole manipulée, Paris, La Découverte/Poche, 2000, 221 p.

BULINGE F., «Méthode globale d'analyse d'information », in Managementstratégique de l'information (sous la dir. de F. BULINGE), Paris, éditions WEKA, juin 2010, 40 p.

BULINGE F., "Rumeurs et attaques informationnelles sur Internet ", Cabiers de la sécurité, n ${ }^{\circ} 6$, octobre-décembre 2008, Institut des hautes études de sécurité, La Documentation française, pp.34-41

CIALDINI R., Influence et manipulation: Comprendre et Maîtriser les mécanismes et les techniques de persuasion, Paris, First Éditions, 2004, 318 p.

COURBET D. et al., «Les effets persuasifs de l'E-publicité perçue « sans conscience » en vision périphérique, implications pour les recherches sur la réception des médias ", Questions de communication, $\mathrm{n}^{\circ} 14$ (2008), pp. 197-219

FRANCART L., La guerre du sens, pourquoi et comment agir sur les champs psychologiques, Paris, Économica, 2000, 311 p.

GUEGUEN N., Psychologie de la manipulation et de la soumission, Paris, Dunod, 2002, $303 \mathrm{p}$.

JOULE R.-V., BEAUVOIS J.-L., Petit traité de manipulation à l'usage des honnêtes gens, Grenoble, PUG, 2002, 286 p.

Livre blanc sur la sécurité intérieure, La Documentation française, 2006.

Livre blanc sur la défense et la sécurité, La Documentation française, 2008.

MUCCHIELLI R., La subversion, Paris, éditions CLC, 1976, 184 p.

PERLOFF R., The Dynamics of Persuasion, Communication and Attitudes in the 21st Century, London, Lawrence Erlbaum Associates, 2003, 392 p.

PESSIGLIONE M. et al., Subliminal Instrumental Conditioning Demonstrated in the Human Brain, Neuron n59, August 28, 2008, p. 561-567.

SALMON C., Storytelling, la machine à fabriquer des histoires et à formater les esprits, Paris, La Découverte, 2007, 239 p.

TCHAKHOTINE S., Le viol des foules par la propagande politique, 1939, éditions Gallimard, 1992, 605 p. 
CASTEL P.-H., « Le passage à l'acte ", in Dictionnaire de la violence, sous la direction de MARZANO M., PUF, 2011.

Mouvements islamistes radicaux - terrorisme - jihadisme

AKMOUCHE W., HEMERY H., « La propagande jihadiste sur Internet : diagnostic et perspectives ", Les Cabiers de la sécurité, n \% , octobre-décembre 2008, p.53-58

BAUDRILLARD J., Power inferno, Paris Éditions Galilée, 2002, 82 p.

CASONI D., BRUNET L., "Philosophie groupale et action terroriste », in CASONI, BRUNET (dir.), Comprendre l'acte terroriste, Presses universitaires du Québec, 2003, p. $75-92$

KEPEL G. (Dir.), Al-Qaïda dans le texte, Paris, Puf, 2005, 440 p.

L'HEUILLET H., Aux sources du terrorisme, de la petite guerre aux attentats suicides, Fayard, 2009, 346 p.

LANDAU P., Pour Allah jusqu'à la mort, éditions du Rocher, 2008, 298 p.

MALLET J.-C., Défense et sécurité nationale : le livre blanc, Odile Jacob, La Documentation française, 2008, 336 p.

NEYRAT F., Le terrorisme, la tentation de l'abìme, Larousse, 2009, 223 p.

ROUGIER B. (dir.), Qu'est-ce que le salafisme? Puf, 2008, 271 p.

SGDN, La France face au terrorisme Livre blanc du Gouvernement sur la sécurité intérieure face au terrorisme, La Documentation française, 2006, 144 p.

THOMAS D., "Le rôle d'internet dans la diffusion de la doctrine salafiste ", in ROUGIER, Qu'est-ce que le salafisme? Puf, 2008, p. 87-102.

Résumé : Cet article est le fruit d'une recherche effectuée en 2008 sous l'égide de l'Institut national des hautes études de sécurité et de justice (INHESJ). Elle portait sur les techniques de manipulation employées sur Internet par des mouvements radicaux, notamment islamistes, et leurs conséquences possibles sur les internautes. À partir des données théoriques de la littérature, nous avons élaboré une grille d'analyse permettant de mesurer le niveau de manipulation de divers supports-contenus disponibles sur Internet et le risque lié à l'effet d'exposition possible sur un public supposé vulnérable.

Mots-clés : Influence, manipulation, subversion, analyse de renseignement, groupes radicaux.

Abstract : This article is the result of a research conducted in 2008 under the auspices of the National Institute of Advanced Studies on Security and Justice (INHESJ). It focused on manipulation techniques used on the Internet by radical movements, including Islamists, and their potential impact on users. From the theoretical data from the literature, we have developed an analytical matrix to measure the level of manipulation of various objects belonging to the islamist web nebula and the risk related to the "effect of possible exposure" applied to a public supposed to be vulnerable.

Keywords : Influence, Manipulation, Subversion, Intelligence analysis, Islamism. 